\title{
Low-cost, widespread and reproducible mold fabrication technique for PDMS-based microfluidic photonic systems
}

\author{
Szymon Baczyński $^{* 1}$, Piotr Sobotka ${ }^{1}$, Kasper Marchlewicz ${ }^{2}$, Artur Dybko $^{2}$, and Katarzyna Rutkowska ${ }^{1}$ \\ ${ }^{1}$ Faculty of Physics, Warsaw University of Technology, Koszykowa 75, 00-662 Warszawa, \\ ${ }^{2}$ Faculty of Chemistry, Warsaw University of Technology, Noakowskiego 3, 00-664 Warszawa
}

Received March 17, 2020; accepted March 27, 2020; published March 31, 2020

\begin{abstract}
In this letter the possibility of low-cost fabrication of molds for PDMS-based photonic microstructures is considered. For this purpose, three different commercially available techniques, namely UVcuring of the capillary film, 3D SLA printing and micromilling, have been analyzed. Obtained results have been compared in terms of prototyping time, quality, repeatability, and re-use of the mold for PDMS-based microstructures fabrication. Prospective use for photonic systems, especially optofluidic ones infiltrated with liquid crystalline materials, have been commented.
\end{abstract}

Poly(dimethylsiloxane) (PDMS) is a biostable synthetic polymeric material with specific elastic and rheological properties. These features, combined with nontoxicity, biocompatibility, blood compatibility, and durability, make this elastomer widely used in modern technology [1]. In addition, PDMS is chemically inert, thermally stable, permeable to gases, simple to manipulate, characterized by an inexpensive fabrication process, and can conform to submicron features to develop complex microstructures [2], including those for Lab on a Chip and Photonic Lab on a Chip [3]. In the context of optical applications, PDMS possesses even more advantageous properties, such as: a refractive index of about 1.41 @ $589 \mathrm{~nm}$ [4], high dielectric constant and breakdown field, isotropy and homogeneity, as well as high optical transparency in the UV, VIS and NIR spectral ranges [5].

All these features make PDMS a benign, beneficial and profitable material to be applied in many research facilities worldwide, reviewed in a considerable number of publications appearing annually, in which new, hightech applications of PDMS microstructures are shown. Through biocompatibility and permeable to gases, PDMS is widely used in chemical and clinical analysis, smallscale organic synthesis, biomedical and implantable devices, and biomimetic systems [6]. For this purpose, such structures as microreactors, microchips for capillary gel electrophoresis, and hydrophobic vent valves have been successfully fabricated [7]. Regarding photonic systems, including those dedicated to optofluidics, optical MEMS [8], microlenses [9] or electrical tuning of the LC: PDMS channels [10] have been realized in PDMS-based microstructures.

One of the most popular methods to reproduce the micropatterns in polymer materials such as PDMS is the

*E-mail: szymon.baczynski.dokt@pw.edu.pl replica-molding technique. The latter provides great capabilities to pattern PDMS at the microscale even in low-resource or educational laboratories. However, one should keep it in mind that geometrical resolution is a serious limiting parameter in developing practical platforms. In the case of photonic systems, when highresolution is required, photolithographic processes are still preferably applied to obtain the best-quality master molds. Soft lithography with photoresist (e.g. SU-8) applied to transfer a specific pattern onto the mold substrate through a photomask is typically associated with high costs. Despite the price and long processing time, the SU-8 molds have the best quality, resolution and appropriate wall texture. Keeping in mind that the goal of rapid prototyping is to reduce the costs and time to create a mold, while maintaining quality and high resolution [6], the application of new photoresists and masks allows for soft lithography to become cheaper, taking up to few hours to create the stamp. In parallel, different strategies for simple and easily accessible solutions are employed to create low-cost molds of satisfying quality. Apart from the above-mentioned photolithography, other basic methods of creating planar systems can be distinguished which are e.g.: (i) use of thermoshrinkable polymers or UV-cured photostencil films, (ii) 3D printing, or (iii) creating a negative or positive mold in plastic or polymers with the use of a micromilling machine or laser. All of them are considered as greatly limiting the costs and time of the mold fabrication. This is particularly the case of the capillary film usage, where the whole process is cheap and easy, taking, in total, less than 1 hour [11]. On the other hand, 3D printing is becoming more and more popular every year, with many scientific groups carrying out their research using various types of 3D printing methods. 3D printing techniques include: Stereolithography (SLA), Multi Jet Modeling (MJM), Fused Deposition Modeling (FDM) and Selective Laser Sintering (SLS). In each of them, different types of devices can be distinguished depending on the components applied, giving thus great opportunities for testing and choosing the optimal equipment for a specific application. 3D printing seems to be a promising strategy for mold fabrication for microfluidic systems thanks to low-cost setups, rapid prototyping and 3D digital design 
[12]. Additionally, micromilling and laser ablation are other methods for molds fabrication to be applied in PDMS-based microsystems. They involve the creation of planar structures in e.g. PMMA or other materials. According to [13], micromilling is a relatively simple technique and can produce channel features down to 50 $\mu \mathrm{m}$, which is enough for most microfluidic applications. Laser ablation is also a good choice as an inexpensive fabrication method for prototyping, preceding the manufacturing of highly expensive and time-consuming molds with e.g. photolithographic techniques. The fabrication of microfluidic components made by femtosecond laser ablation has been presented in [14]. Further research on finding low-cost methods that at the same time guarantee high resolution, good repeatability and perfect quality molds for demanding applications still stands. The comparison of different methods, specifically devoted to non-planar PDMS microfluidic systems, can be found in [15].

In this letter low-cost mold prototyping methods are introduced and compared. Specifically, due to a multitude of possibilities, investigations shown here are limited to three different methods, which are molding forms fabrication: (i) with the use of PHAT (translucent) film; (ii) by $3 \mathrm{D}$ printing, and (iii) by milling in plastic.

Translucent films, known also as capillary or photostencil films, of various thicknesses from 50 to 200 microns, are widely used in biochemistry to create molds thanks to their simple design and manufacturing process [10]. The mask needed to devolve a pattern on the film can be easily prepared with the use of CorelDraw or AutoCAD software and then printed on a transparency plotter film. In this way, many masks can be printed on one piece of the sheet, reducing the costs of the whole process. The prepared mask (negative) is applied on the capillary film (here Chromaline Super PHAT 200 from IKONICS Corp.), which is firstly appropriately placed on a glass substrate. The structure prepared in this way is placed under a UV lamp and only the area where the mask is transparent is hardened by UV radiation. After an appropriate curing time of about 2 minutes, the mask is removed. Then, the uncured capillary film is carefully washed away with water. The final stage of the fabrication process is drying the mold. The whole fabrication process takes up to 1 hour. It is very simple and cheap, which makes it considered a widely available and quick prototyping method. The only disadvantage is the nonrecurring mold with an additional, only relatively simple pattern which is to be reproduced. What is more, the repeatability of this method is awkward with many deviations affecting the mold features at any stage of the fabrication process. As shown in Fig. 1, the quality of the mold obtained with the use of the UV-cured photostencil film is rather poor.

As one can notice, there are areas where the capillary film is not properly rinsed or has been subjected to UV exposure for too long. However, the probability of making a good quality mold is sufficient to conclude that this method is noteworthy. There are also additional aspects such as low production cost and short prototyping time, which are worth considering when choosing this method.

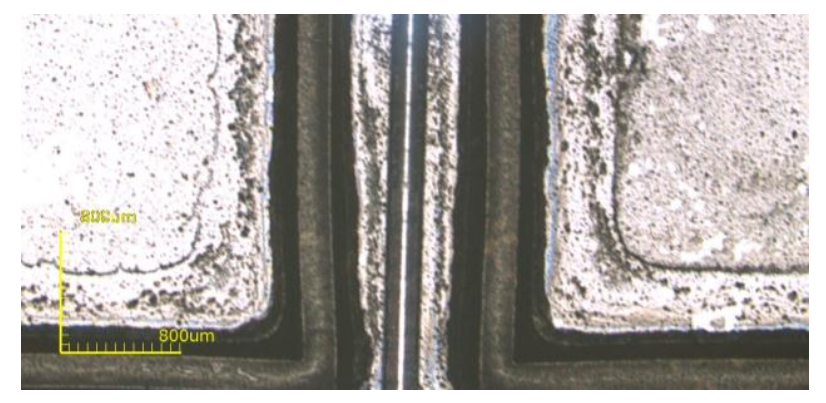

Fig. 1. Capillary film mold with optical polymer fiber applied additionally to obtain the main central channel in the mold (photo made with the use of Olympus LEXT OLS3100 microscope).

Another method for mold fabrication which has been considered in this work as a dynamically developing technology is 3D printing. Specifically, the SLA 3D printing applied here, works on the principle of spot curing of a special resin. Layer by layer, the model is pulled out of the bath with a special arm. Every year there are new models of this kind of printers using laser diodes with higher power and better resolution, improving thus their performance. This allows for more accurate prints at similar costs as for earlier models of printers. For the 3D printer used in this work, which was the Formlabs Form 2 with Standard Clear (RS-F2-GPCL-04) resin, some preliminary tests have ended up with the following parameters: a layer thickness of $25 \mu \mathrm{m}$ and laser spot size of about $140 \mu \mathrm{m}$ in diameter. Anyhow, the mold design and fabrication is a little more complicated than when capillary film is used. Before printing the mold with a $3 \mathrm{D}$ printer, many tests have to be carried out. Despite the parameters of the 3D printer itself, the application of specific resin often causes problems during the printing process, including model peeling off from the printer base, drying excess resin or difficulties in washing out excess resin. After testing the printing process, one can proceed to the appropriate part of the mold design. The 3D model of the mold has been eventually made in AutoCAD software, taking into account the corrections from the previous printing tests. A properly prepared STL ("StereoLithography") file has been uploaded to the 3D printer. After a few hours of printing with the highest resolution available, the mold has been washed with isopropyl alcohol. The whole process of 3D printing is relatively fast, although it takes longer than the firstly mentioned method based on capillary film. The quality of the mold obtained from the 3D printer can be evaluated by analysing the photos presented in Fig. 2 . 

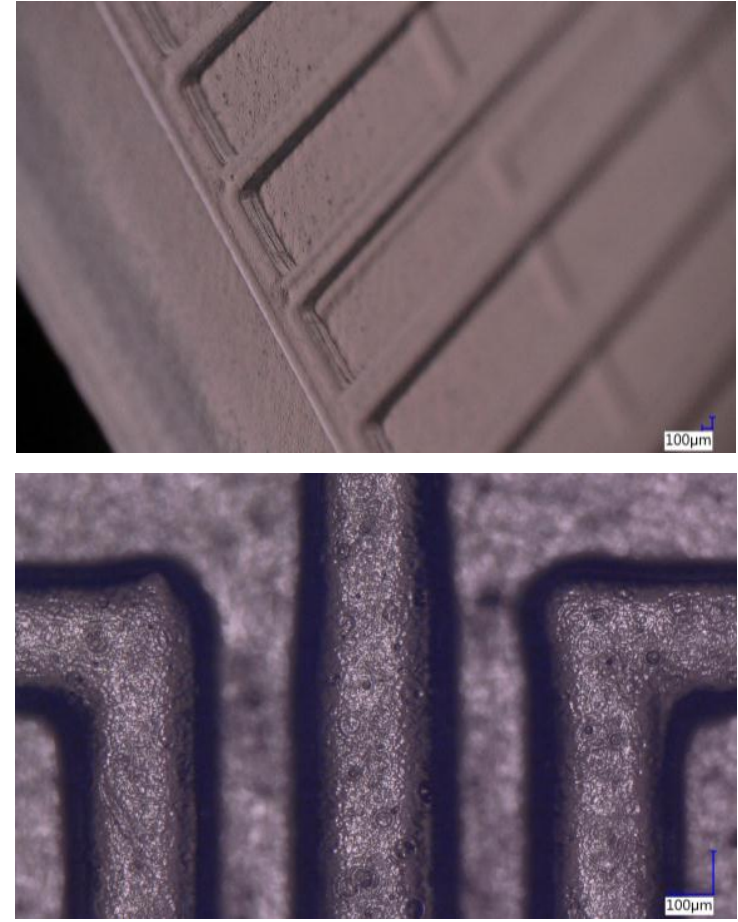

Fig. 2. Photo (top) and high-resolution photo (bottom) of the 3D printed mold obtained with the KEYENCE VHX 5000 digital microscope. The scale is presented in the right-bottom part of each picture.

As it can be seen, the initial test molds made with a 3D printer had some disadvantages. Firstly, the subsequent layers of the resin can be seen on the walls, as an effect of imperfect mechanical parts of the printer. In addition, the upper layer of the mold shows large roughness caused by the shape of the laser beam spot used to cure the resin. Apart from some drawbacks, 3D printing can be still considered as an interesting and suitable method for prototyping molds, while it does not require an experienced laboratory stuff and the whole process is inexpensive and relatively fast.

Eventually, the micromilling in PMMA material can be used for accurate mold prototyping. In this case, a very precise and expensive machine is used, which has to be operated by a qualified person. The whole process of preparing the model is similar to that used for the 3D printing. However, the process of PMMA treatment itself takes up to few hours to obtain a mold. After cleaning it from material residue it can be immediately used. Figure 3 shows that the mold is of very high quality. The walls are almost perpendicular to the base, with no flaws or roughness. The upper layer of the mold shows periodic unevenness, but it seems to be negligible in further application of the mold. Micromilling in PMMA is quite fast but it is not as cheap as other analysed mold prototyping methods for microfluidic systems in PDMS. Comparison of all methods tested in this work can be found in Table 1.

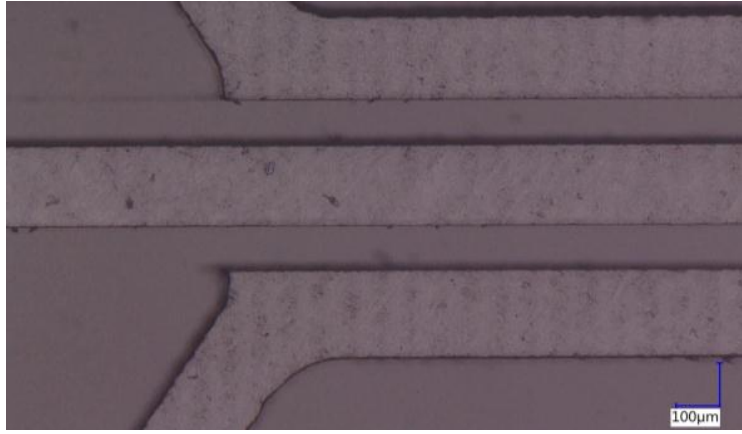

Fig. 3. Micromilled mold (photo made with use of the KEYENCE VHX 5000 digital microscope).

Table 1. Comparison of speed, repeatability, quality and reuse of different fabrication methods for the mold fabrication tested in this work.

\begin{tabular}{|c|c|c|c|c|}
\hline Fabrication method & $\begin{array}{c}\text { Prototyping } \\
\text { time }\end{array}$ & $\begin{array}{c}\text { Repeat- } \\
\text { ability }\end{array}$ & Quality & $\begin{array}{c}\text { Reusable } \\
\text { mold }\end{array}$ \\
\hline $\begin{array}{c}\text { UV-cured capillary } \\
\text { film }\end{array}$ & $<1$ hour & low & good & no \\
\hline 3D printing (SLA) & few hours & good & low & yes \\
\hline Micromilling & few hours & best & best & yes \\
\hline
\end{tabular}

In this letter different methods have been proposed to save the process time and budget, while presenting a procedure for the construction of good-quality molds for microstructured PDMS platforms for photonic applications. Specifically, as summarized in Table 1, a comparison between three different techniques has been made. All presented methods have some advantages over each other, as described in the text. Preliminary tests have shown that micromilling is the best one among the tested methods in producing a low-cost mold of sufficiently high quality. In addition to molds characterization, additional tests in which PDMS is used as a cast on prepared molds have been also made. The evaluation confirming the initial assumptions about the molds quality and the results of the PDMS casts tests will be shown elsewhere. The homeotropic alignment of liquid crystalline molecules on PDMS walls for tentatively infiltrated microstructures was obtained only when the translucent film molds were used.

\section{References}

[1] K. Sangamesh, C.T. Laurencin, M. Deng, Natural and Synthetic Biomedical Polymers (Elsevier, Amsterdam 2004).

[2] A. Mata et. al, Biomed. Microdev. 7(4), 281 (2005).

[3] I. Rodríguez-Ruiz et al., Anal. Chem. 88(13), 6630 (2016).

[4] SYLGARD ${ }^{\mathrm{TM}} 184$ Silicone Elastomer, Technical Data Sheet

[5] N.E. Stankova et al., Appl. Surface Science 374, 96 (2016).

[6] J.C. McDonald et al., Electrophoresis 21(1), 27 (2000).

[7] T. Fujii, Microelectronic Eng. 61, 907 (2002).

[8] F. Schneider et al., Sensors Actuat. A: Physical 151(2), 95 (2009)

[9] T.K. Shih et al., Microelectronic Eng. 83(11-12), 2499 (2006).

[10] K.A. Rutkowska et al., Photon. Lett. Poland 9, 48 (2017).

[11] D. Kalinowska et al., Biosensors Bioelectr. 126, 214 (2019).

[12] N. Bhattacharjee et al., Lab on a Chip 16 (10), 1720 (2016).

[13] I.R.G. Ogilvie et al., J. Micromech. Microeng. 20(6), 065016 (2010).

[14] D. Gomez et al., Opt. Eng. 44(5), 051105 (2005).

[15] Y. Hwang, R.N. Candler, Lab on a Chip 17(23), 3948 (2017). 